\title{
Precoding Design and User Selection for Multibeam Satellite Channels
}

\author{
Ahmad Gharanjik*†, Bhavani Shankar M. R.*, Pantelis-Daniel Arapoglou, ${ }^{\ddagger}$, Mats Bengtsson ${ }^{\dagger}$ and Björn Ottersten*† \\ ${ }^{*}$ Interdisciplinary Centre for Security, Reliability and Trust (SnT), University of Luxembourg \\ ${ }^{\dagger}$ KTH Royal Institute of Technology, Stockholm, Sweden \\ $\ddagger$ Ajilon Aerospace, Noordwijk, Netherlands
}

\begin{abstract}
Precoding for the downlink of a multibeam satellite system has been recently shown, under ideal conditions, to be promising technique towards employing aggressive frequency reuse gainfully. However, time varying phase uncertainties imposed by the components and the channel, combined with delayed feedback perturbs the channel state information at the transmitter (CSIT). In this paper, we consider a power constrained robust formulation of the downlink precoding problem to counter the phase uncertainties. In particular it considers imposing conditions on the average signal to interference plus noise ratio (SINR), to deal with imperfect CSIT. In addition to the robust formulation, the primacy of user selection is highlighted and a new approach exploiting the satellite system design is proposed. Performance of the derived robust precoder in conjunction with the proposed location based user selection is then evaluated and the gains are tabulated.
\end{abstract}

\section{INTRODUCTION}

Demand for increased data due to new applications and services like aeronautical and maritime, machine to machine, broadband internet and interactive multimedia has warranted an investigation of spectrally efficient techniques. While the current generation of multibeam satellites reuse frequencies in a typical four colour pattern [1], the increased demand per beam has motivated a consideration of aggressive frequency reuse [2], [3]. While aggressive reuse enhances the bandwidth, interference from co-channel beams need to be managed. Towards this, downlink precoding techniques have been considered to reduce co-channel interference and achieve gains from full-frequency reuse. A key requirement in implementing an efficient precoding is the availability of perfect CSIT.

Unlike the terrestrial counterparts, GEO satellite communication systems result in long round trip delays (RTD) between the gateway (GW) and User Terminal (UT). In particular, the two hop propagation delay in the GEO orbit is about 250 milliseconds [4] compared to the few milliseconds in cellular systems. In the satellite channel, it was observed that the amplitude of the channel changes slowly compared to the RTD [5], [6]. However, the variations in the phase component of the channel are rapid due to phase noise contribution from the payload and the time dependent channel variations [4], [6]. This time varying nature of the channel coupled with a high RTD result in outdated CSIT, or more specifically outdated channel phase [6].

Towards realizing precoding in practical systems, an impact of the outdated CSIT needs to be undertaken and precoding algorithms robust to uncertainties need to be devised. A robust precoding design for a channel with outdated phase is described in [6] based on probabilistic approach. Therein, the precoder is designed to guarantee the desired quality of service (QoS) goals based on the outage probability for all users. However, there are also several other possible robustness paradigms available [7]. Towards guaranteeing different QoS goals, we consider another approach, namely the expectation based approach. We design a robust precoder that can ensure average SINR of all users are above a predetermined threshold. We also study a heuristic user selection method and discuss its effect on the system performance.

Notation: Boldface upper-case letters denote matrices, boldface lower-case letters denote column vectors. $\mathbf{X} \succcurlyeq 0$ means that matrix is positive semidefinite (PSD). The superscripts $(\cdot)^{\dagger}$ and $(.)^{T}$ denote the Hermitian conjugate, the transpose of a matrix, respectively. The Hadamard product (Schur product) is denoted by $\odot$. If $\mathbf{x}=\left[x_{1}, \ldots, x_{K}\right]^{T}$ is a $K \times 1$ vector, then $\operatorname{diag}(\mathbf{x})$ is a square diagonal matrix with the elements of vector $\mathbf{x}$. Further, for any scalar $a$, $a^{\mathbf{x}}=\left[a^{x_{1}}, a^{x_{2}}, \ldots, a^{x_{K}}\right]^{T}$ and $\mathbf{x}^{a}=\left[x_{1}^{a}, x_{2}^{a}, \ldots, x_{K}^{a}\right]^{T}$.

\section{System And Channel Model}

We consider a typical Ka-band multibeam GEO satellite system with $K$ beams [2], [6] employing a full frequency reuse where all the beams operate at the same frequency. We further assume a single feed per beam scenario with $K$ antenna feeds at the satellite used to form the $K$ fixed beams. Further, each feed has a constraint on the maximum radiated power denoted by $P_{i}$. Towards focussing on the precoder design, a single GW is assumed to manage $K$ adjacent beams and the feederlink (the link between the GW and the satellite) is considered ideal; such assumptions are commonplace in related literature [2], [3]. Time division multiple access (TDMA) is employed on the user downlink (link between satellite and user) wherein a single user is served in a beam for every time slot. The focus of this work relates to the precoding on the forward link (the link from the GW towards users); a preliminary analysis of the return link can be found in [8].

Since the beams are not perfectly isolated, each user receives transmissions from all the $K$ feeds. This combined with the use of TDMA results in a user being interfered by $K-1$ co-channel users. Such a system then resembles the traditional multiuser MISO downlink, thereby facilitating further analysis. Also, in Ka-band scenarios, the channel is typically modelled as being frequency flat.

The time varying downlink channel vector between the $K$ 
satellite transmit antennas and $i^{\text {th }}$ user can be expressed as,

$$
\mathbf{h}_{i}(t)=\sqrt{C}_{i} \mathbf{b}_{i}^{\frac{1}{2}} \odot \mathbf{r}_{i}(t)^{-\frac{1}{2}} \odot e^{\mathrm{j} \boldsymbol{\theta}_{i}(t)}
$$

where $\mathbf{r}_{i}=\left[r_{i 1}, r_{i 2}, \ldots, r_{i K}\right]^{T}$ represents the time varying rain attenuation (for simplicity we drop $t$ ). The elements of $\mathbf{r}_{i}$ in $\mathrm{dB}, 10 \log _{10}\left(r_{i k}\right), 1 \leq k \leq K$, have a lognormal distribution [9], [10]. $\boldsymbol{\theta}_{i}(t)$ is a $K \times 1$ vector that represents the phase components of the channel. We let $\boldsymbol{\theta}_{i}(t) \triangleq\left[\theta_{i, 1}(t), \theta_{i, 2}(t), \ldots, \theta_{i, K}(t)\right]^{T} .\left[\mathbf{b}_{i}\right]_{k}$ denote the beam gain from the $k^{\text {th }}$ antenna to $i^{\text {th }}$ user and $C_{i}$ is a scaling parameter derived from link analysis, the details of which are mentioned in Section IV. Note that, the amplitude of the channel is also affected by cloud attenuation and the gaseous absorbtion [5], [11]. However, since these components are negligible compared to the rain attenuation in Ka-band and change slower than the rain attenuation, they are not considered in (1).

Based on (1), the baseband time varying sub-channel in the forward-link between the antenna $j$ and the user $i$ at time $t$ is denoted by $h_{i, j}(t)=\left|h_{i, j}\right| e^{\mathrm{j} \theta_{i, j}(t)}$. As mentioned, the amplitude of this channel is dominated by the rain attenuation which changes negligibly in the RTD interval. Therefore, the temporal variations of the amplitude are considered to be negligible over the intervals of interest and hence time dependence is omitted from $\left|h_{i, j}\right|$.

On the other hand, the phase of the sub-channels can be affected by a number of time varying components. For example, each feed will contribute a random phase component to the sub-channels; this arise from the use of different local oscillators (LO) on-board the satellite for each feed. This random phase component is also called phase noise of the LO. Further, the rain, cloud and gaseous absorbtion can introduce time varying phase variations to each sub-channel; such variations are faster than those for amplitude. These time varying phase components are independent across the subchannels and are incorporated in the channel model as $\theta_{i, j}(t)$.

The $i^{\text {th }} \mathrm{UT}$ estimates the amplitude and the phase of each of the sub-channels at time $t_{0}$ and feeds them back to the GW. As mentioned earlier, due to the long delay in GEO satellite system and time phase components, the phase of the channel when precoding is applied at $t_{1} \approx t_{0}+250 \mathrm{~ms}$ will be different than $\boldsymbol{\theta}_{i}\left(t_{0}\right)$. Since $\theta_{i, j}\left(t_{1}\right)$ is the actual phase for the sub-channel $h_{i, j}\left(t_{1}\right)$ and further using $\boldsymbol{\theta}_{i}\left(t_{1}\right)$, we model the temporal variations as,

$$
\boldsymbol{\theta}_{i}\left(t_{1}\right)=\boldsymbol{\theta}_{i}\left(t_{0}\right)+\mathbf{e}_{i},
$$

where $\mathbf{e}_{i} \triangleq\left[\mathrm{e}_{i, 1}, \mathrm{e}_{i, 2}, \ldots, \mathrm{e}_{i, K}\right]^{T}$ is the phase error, or phase uncertainty, vector with independnt identically dustributed (i.i.d) Gaussian random entries, $\mathbf{e}_{i} \sim \mathcal{N}\left(\mathbf{0}, \sigma_{i}^{2} \mathbf{I}\right)$. Here, $\sigma_{i}^{2}$ is the variance of the phase error for the $i^{\text {th }}$ user. For ease of notation, we define the corresponding channel at $t_{1}$ and $t_{0}$ by $\mathbf{h}_{i}$ and $\widehat{\mathbf{h}}_{i}$, respectively. Under these notations and assuming that the channel amplitudes are identical at $t_{1}$ and $t_{0}$, the $K \times 1$ channel fading coefficients from all antenna feeds towards the $i^{\text {th }} \mathrm{UT}$ at instance $t_{1}$ are then given by

$$
\mathbf{h}_{i}=\widehat{\mathbf{h}}_{i} \odot \mathbf{q}_{i}
$$

where $\mathbf{q}_{i}=e^{\mathbf{j} \mathbf{e}_{i}}$. In (3), $\widehat{\mathbf{h}}_{i}$ is a known channel vector at $t_{0}$, but $\mathbf{q}_{i}$ is a random vector due to the randomness of $\mathbf{e}_{i}$ which represent the uncertainty. We further assume that the correlation matrix of $\mathbf{q}_{i}$, denoted by $\mathbf{C}_{i}$, is known at $\mathrm{GW}$ and takes the form,

$$
\mathbf{C}_{i} \triangleq \mathrm{E}\left\{\mathbf{q}_{i} \mathbf{q}_{i}^{\dagger}\right\}=\mathrm{E}\left\{\mathbf{Q}_{i}\right\}
$$

where $\mathbf{Q}_{i} \triangleq \mathbf{q}_{i} \mathbf{q}_{i}^{\dagger}$ which is a random matrix. The diagonal elements of $\mathbf{C}_{i}$ are unity and off-diagonal entries can be found by using the moment generating function of the Guassian random variable as follows,

$$
\begin{aligned}
{\left[\mathbf{C}_{i}\right]_{l m} } & =\mathrm{E}\left\{\left[\mathbf{Q}_{i}\right]_{l m}\right\}=\mathrm{E}\left\{e^{\mathrm{je}_{i, l}}\right\} \mathrm{E}\left\{e^{-\mathrm{je}_{i, m}}\right\} \\
& =e^{-\sigma_{i}^{2} \triangleq \rho_{i} .}
\end{aligned}
$$

\section{SYSTEM DESIGN}

\section{A. Precoding Formulation}

The complex signal intended for $i^{\text {th }}$ user is weighted at the GW by the corresponding precoding vector $\mathbf{w}_{i} \in \mathbb{C}^{K}$, and are multiplexed and transmitted from the GW to the involved transparent satellite (amplify and forward). Ideal feeder link and lossless processing on-board the satellite are assumed [6]. Then, the received SINR at $i^{\text {th }}$ user takes the form,

$$
\mathrm{SINR}_{i}=\frac{\operatorname{Tr}\left(\mathbf{R}_{i} \mathbf{W}_{i}\right)}{\sum_{j \neq i} \operatorname{Tr}\left(\mathbf{R}_{i} \mathbf{W}_{j}\right)+N_{0}},
$$

where,

$$
\mathbf{R}_{i} \triangleq \mathbf{h}_{i} \mathbf{h}_{i}^{\dagger}=\operatorname{diag}\left(\widehat{\mathbf{h}}_{i}\right) \mathbf{Q}_{i} \operatorname{diag}\left(\widehat{\mathbf{h}}_{i}^{\dagger}\right),
$$

$\mathbf{W}_{i} \triangleq \mathbf{w}_{i} \mathbf{w}_{i}^{\dagger}$ and $\mathbf{R}_{i} \in \mathbb{C}^{K \times K}$ is the instantaneous channel correlation matrix at $t_{1} . N_{0}$ is the variance of the additive receiver noise at UT. In (7), $\widehat{\mathbf{h}}_{i}$ is the known (estimated) channel vector at $t_{0}$ and $\mathbf{Q}_{i}$ is a random matrix which represents the phase uncertainty and makes the SINR a random variable. Therefore, in this paper, we define the required QoS goals based on the average performance of the system. Since the average SINR of the users is closely related to the average performance of the system, we are motivated to use $\mathrm{E}\left\{\mathrm{SINR}_{i}\right\}$ as a QoS measure. To this end, the precoder is designed to satisfy the requirement on the average SINR, namely $\mathrm{E}\left\{\mathrm{SINR}_{i}\right\} \geq \gamma_{\mathrm{th}}$, where expectation is over the random $\mathbf{q}_{i}$.

Following this approach, the robust precoding formulation can be written as,

$$
\begin{array}{|cl}
\mathcal{G}: \underset{\mathbf{W}, \tau_{1}, \ldots, \tau_{K}}{\operatorname{minimize}} & \sum_{i=1}^{K} \tau_{i} P_{i} \\
\underset{\forall i}{\text { subject to }} & \mathrm{E}\left\{\mathrm{SINR}_{i}\right\} \geq \gamma_{\mathrm{th}}, \\
& {\left[\sum_{j=1}^{K} \mathbf{W}_{j}\right]_{i, i} \leq \tau_{i} P_{i}, \tau_{i} \leq 1,} \\
& \mathbf{W}_{i} \succcurlyeq 0, \operatorname{rank}\left(\mathbf{W}_{i}\right)=1 .
\end{array}
$$

where $\mathbf{W} \triangleq\left\{\mathbf{W}_{1}, \ldots, \mathbf{W}_{K}\right\}$. In this formulation, $P_{i}$ denotes the transmit power constraint of the $i^{\text {th }}$ antenna feed where the $i^{\text {th }}$ user is located and served in a beam formed by $i^{\text {th }}$ antenna. Here, $\tau_{i}$ is the power factor similar to one introduced in [12]. Since each antenna has its own amplifier, $\tau_{i}$ can control the transmit power of each antenna feed. On-board the satellite, 
the transmitted power is a scarce resource. Hence the objective of this optimization problem is to minimize the total transmit power subject to requirements on average SINR for each user with the lowest possible transmit power from each antenna feed. In this formulation, the rank one constraint is non-convex, but can be relaxed by retaining only the semidefiniteness constraint $\mathbf{W}_{i} \succcurlyeq 0$, which is a convex constraint. Later, we will discuss how to extract rank one solutions from $\mathbf{W}_{i} \mathbf{s}$.

In general, it is difficult to evaluate the exact value of $\mathrm{E}\left\{\mathrm{SINR}_{i}\right\}$, since the expectation of $\mathrm{SINR}_{i}$ over $\mathbf{q}_{i}$ is untractable. We use an approximation for $\mathrm{SINR}_{i}$ denoted by $\operatorname{SINR}_{i}^{\prime}$ where the received signal and interference power are replaced by their expected values [13], [14]. Specifically, $\mathrm{SINR}_{i}^{\prime}$ can be expressed as,

$$
\mathrm{SINR}_{i}^{\prime} \triangleq \frac{\mathrm{E}\left\{\operatorname{Tr}\left(\mathbf{R}_{i} \mathbf{W}_{i}\right)\right\}}{\mathrm{E}\left\{\sum_{j \neq i} \operatorname{Tr}\left(\mathbf{R}_{i} \mathbf{W}_{j}\right)\right\}+N_{0}} .
$$

Numerical results show that $\mathrm{SINR}_{i}^{\prime}$ is a tight lower bound on $\mathrm{E}\left\{\mathrm{SINR}_{i}\right\}$. Therefore, if the precoding matrices satisfy the constraint $\operatorname{SINR}_{i}^{\prime} \geq \gamma_{\text {th }}$, then they can also satisfy the constraint $\mathrm{E}\left\{\mathrm{SINR}_{i}\right\} \geq \gamma_{\text {th }}$. So, we can replace the constraint on the average $\operatorname{SINR}$ in $\mathcal{G}$ by the constraint $\operatorname{SINR}_{i}^{\prime} \geq \gamma_{\text {th }}$.

The new constraint can be rewritten as,

$$
\operatorname{Tr}\left(\mathrm{E}\left\{\mathbf{R}_{i}\right\} \mathbf{W}_{i}\right)-\gamma_{\text {th }} \sum_{j \neq i} \operatorname{Tr}\left(\mathrm{E}\left\{\mathbf{R}_{i}\right\} \mathbf{W}_{j}\right) \geq \gamma_{\mathrm{th}} N_{0} .
$$

As can be seen, the expression in (8) can also be interpreted as the result of using long-term (averaging over phase noise) channel correlation matrix, $\mathrm{E}\left\{\mathbf{R}_{i}\right\}$, instead of instantaneous $\mathbf{R}_{i}$, to achieve the robustness. Using (4) and (7), E $\left\{\mathbf{R}_{i}\right\}$ is,

$$
\mathbf{R}_{i}^{\prime}=\mathrm{E}\left\{\mathbf{R}_{i}\right\}=\operatorname{diag}\left(\widehat{\mathbf{h}}_{i}\right) \mathbf{C}_{i} \operatorname{diag}\left(\widehat{\mathbf{h}}_{i}^{\dagger}\right)
$$

Then, by defining $\mathbf{Z}_{i}=\mathbf{W}_{i}-\gamma_{\text {th }} \sum_{j \neq i} \mathbf{W}_{j}$, (9) can be written as,

$$
\operatorname{Tr}\left(\mathbf{R}_{i}^{\prime} \mathbf{Z}_{i}\right)-\gamma_{\mathrm{th}} N_{0} \geq 0,
$$

This is a convex (affine in $\mathbf{W}_{i}$ ) constraint and the effect of the phase uncertainty is reflected in $\mathbf{C}_{i}$. Finally, the expectation based robust optimization problem can be written as,

$$
\begin{array}{|cl|}
\hline \mathcal{G}_{1}: \underset{\mathbf{W}, \tau_{1}, \ldots, \tau_{K}}{\operatorname{minimize}} \\
\begin{array}{c}
\text { subject to } \\
\forall i
\end{array} & \sum_{i=1}^{K} \tau_{i} P_{i} \\
& \operatorname{Tr}\left(\mathbf{R}_{i}^{\prime} \mathbf{Z}_{i}\right)-\gamma_{\mathrm{th}} N_{0} \geq 0, \\
& {\left[\sum_{j=1}^{K} \mathbf{W}_{j}\right]_{i, i} \leq \tau_{i} P_{i}, \tau_{i} \leq 1,} \\
& \mathbf{W}_{i} \succcurlyeq 0 .
\end{array}
$$

In this formulation, the constraints are convex, so it can be solved by using standard convex solvers like CVX [15]. We denote the resulting precoding matrix of $\mathcal{G}_{1}$ by $\mathbf{W}^{\star}=$ $\left[\mathbf{w}_{1}^{\star}, \cdots, \mathbf{w}_{K}^{\star}\right]$ where $\mathbf{w}_{i}^{\star}$ is the precoding vector for the $i^{\text {th }}$ user.

The solution to $\mathcal{G}_{1}$ can yield non-unity rank $\left\{\mathbf{W}_{i}\right\}_{1}^{K}$. In such situations, rank one approximation techniques [16] needs to be employed. Additional details are provided in Section IV regarding the rank one solution of the optimization problem.

\section{B. User Selection}

As mentioned in Section II, it is assumed that a single user is served per beam in a transmission slot; therefore a total of 7 users, one per beam, is targeted. Fig. 1 shows the relative locations of 7 beams. A single user is selected from each beam to make a group of 7 users, and the precoding matrix is subsequently designed. The mode of selecting the users to be served in the same time slot is an important issue that can affect the total performance of the system. In the following, we will introduce a heuristic approach for user selection (US).

The simplest and well known approach is random user selection, where users are simply selected randomly, one from each beam, to form a group of 7 users. Therefore, it is possible that some users with good channel condition (interference) are grouped with some users with bad channel condition. This issue will affect on the feasibility of the problem.

The proposed approach is to select the users based on the level of the interference they receive from other beams before the precoding. In other words, users with low received interference are grouped together and it is the same for users with high interference. The motivation behind this approach is to form groups of users such that they can not adversely affect the feasibility of the precoding design. In a multibeam satellite system, the level of the interference is highly dependent on the location of the users, e.g. users close to the center of the beams receive lower interference compared to the users in the border of the beams. So, we can group users based on their distance form the center of their corresponding beams. We call this approach location based user selection.

Note, that in this approach, it is assumed that location of the users are available at the GW since user terminals are generally fixed. In the next section, effects of user selection on the system performance will be discussed.

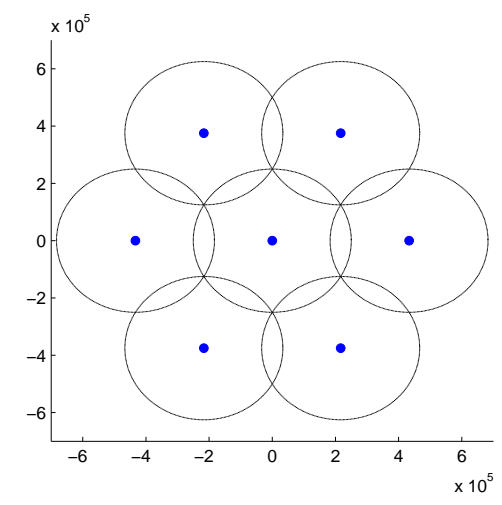

Fig. 1. Locations of 7 beams ( beam radius is $250 \mathrm{Km}$ )

\section{NumERICAL RESUltS}

For the numerical evaluation, we generate 1000 random user location for each of the 7 beams. The users are distributed uniformly within the beams. Based on the model explained in Section II, channel instances are then generated. Given the $i^{\text {th }}$ user's random location within a beam, we define the angle subtended by the chord between $i^{\text {th }}$ user and the $k^{\text {th }}$ beam center at the satellite as $\varphi_{k, i}$. The $3 \mathrm{~dB}$ angle for the $k^{\text {th }}$ beam 
TABLE I. LINK BUdGET AND SySTEM PARAMETERS

\begin{tabular}{c|c} 
Parameter & Value \\
\hline Orbit & GEO, $d_{0}=35786(\mathrm{Km})$ \\
Downlink Band & Ka-Band, $f=20(\mathrm{GHz})$ \\
Number of beams & $K=7$ \\
Beam radius & $250(\mathrm{Km})$ \\
Boltzmann's constant & $\kappa=1.38 \times 10^{-23}(\mathrm{~J} / \mathrm{m})$ \\
Noise bandwidth & $B=50(\mathrm{MHz})$ \\
Satellite antennae gain & $G_{s}, k=38(\mathrm{dBi})$ \\
Receiver gain to noise temperature & $G_{r, i} / T=15(\mathrm{~dB} / \mathrm{K})$ \\
3dB Angle & $\theta_{3 \mathrm{~dB}}=0.4^{\circ}$ \\
TWTA RF Power @ Saturation & $P_{i}=200(\mathrm{~W})$ \\
\hline
\end{tabular}

as $\varphi_{k 3 \mathrm{~dB}}$ which is a constant. The beam gain from the $k^{\text {th }}$ antenna to $i^{\text {th }}$ user is approximated by [17],

$$
\left[\mathbf{b}_{i}\right]_{k}=G_{s, k}\left(\frac{J_{1}\left(u_{k}\right)}{2 u_{k}}+36 \frac{J_{3}\left(u_{k}\right)}{u_{k}^{3}}\right)^{2},
$$

where $G_{s, k}$ is the satellite transmit antenna gain for the $k^{\text {th }}$ beam and $u_{k}=2.07123 \frac{\sin \left(\varphi_{k, i}\right)}{\sin \left(\varphi_{k 3 \mathrm{~dB}}\right)}$. Here, $J_{1}$ and $J_{3}$, respectively, are the first and third order Bessel functions of first kind. In (1), the coefficient $C_{i}$ is defined as,

$$
C_{i}=\left(\frac{\nu}{4 \pi f d_{0}}\right)^{2} \frac{G_{r, i}}{\kappa B T},
$$

to include effects of the free space loss, $\left(\nu /\left(4 \pi f d_{0}\right)\right)^{2}$, UT's receive gain, $G_{r, i}$, and noise power at receiver, $\kappa B T$. We normalized the noise power by $\kappa B T$, so noise power can be assumed to be one. In (13), $\nu$ is the speed of light, $f$ is operating frequency of the downlink, $\kappa$ is Boltzmann's constant, $B$ is noise bandwidth and $T$ is the receive noise temperature.

The system parameters which are used for the channel realization are shown in Table I. Then, considering different US methods, the optimization problem $\mathcal{G}_{1}$ is solved. Let $\mathbf{W}^{\star}=\left\{\mathbf{W}_{1}^{\star}, \ldots, \mathbf{W}_{K}^{\star}\right\}$ denote the solution of the rank one relaxed optimization problem. If $\mathbf{W}_{i}^{\star}$ is rank one, then we can write $\mathbf{W}_{i}^{\star}=\mathbf{w}_{i}^{\star} \mathbf{w}_{i}^{\star \dagger}$ where $\mathbf{w}_{i}^{\star}$ is the optimal solution. Based on numerical evaluations, interestingly, it was observed that $\mathcal{G}_{1}$ yields rank one solution for all feasible realizations.

Fig. 2 shows the feasibility rate of $\mathcal{G}_{1}$ for different phase uncertainty and different US methods. As expected, the lower phase uncertainty results in a higher feasibility rate for both US methods. It is can be seen that for low $\gamma_{\text {th }}$, random US has higher feasibility than location based US. The reason is that in the random US good and bad users (in the sense of received interference) can be grouped together. Hence good users can help bad users to have higher SINR by allowing corresponding antenna feed of the bad user to transmit with higher power and tolerating higher interference from other beams. But, for high $\gamma_{\text {th }}$, good users can no longer help bad user by accepting additional interference, since they themselves need to satisfy high $\gamma_{\text {th }}$. Therefore, it can be observed that for the higher $\gamma_{\text {th }}$, feasibility rate of the location based US is higher than that for random US.

Fig. 3 shows the average total transmitted power for the different amount of phase uncertainty and different US methods. It can be seen that for the medium and high $\gamma_{\text {th }}$, location based US requires lower power compared to the random US. Also, it is observed that transmitted power is not sensitive to

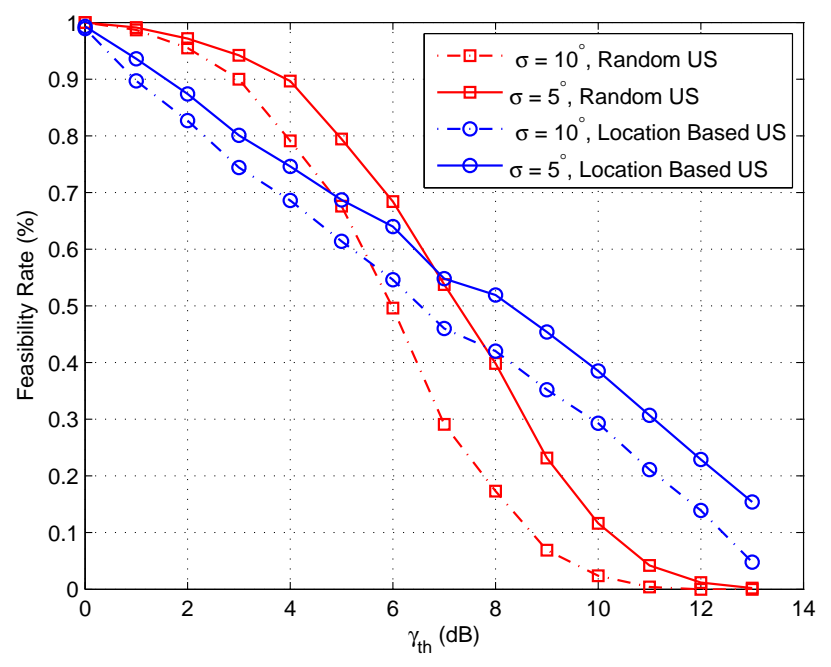

Fig. 2. Feasibility rate of the optimization problem for 1000 channel realizations

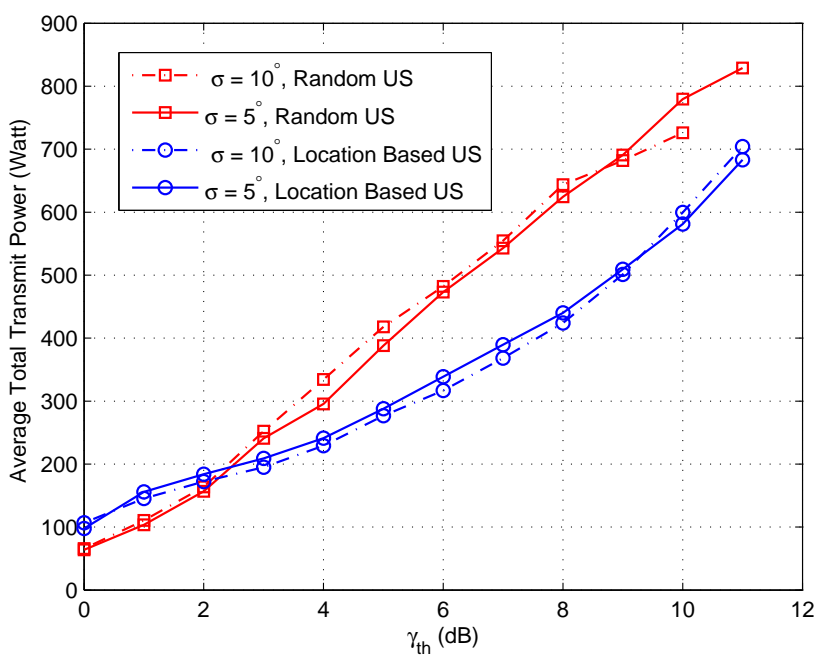

Fig. 3. Dependence of Average Total Transmit power on the Threshold $\gamma_{\text {th }}$

the phase uncertainty since for different amount of the phase uncertainty, variation in the total transmit power is negligible.

From Fig. 2 and Fig. 3, it can be concluded that for lower $\gamma_{\text {th }}$, random US is a better choice since for a lower transmit power, it provides a higher feasibility rate. But, for higher $\gamma_{\text {th }}$, location based US is a better choice while it can provide higher feasibility rate with lower transmit power compared to the random US.

\section{CONCLUSION}

This paper deals with the downlink precoding problem in a multibeam satellite system. It takes into account the outdated channel state information at the transmitter arising due to phase uncertainties introduced by payload and propagation. A robust precoding design is presented, where in conditions on the average SINR is introduced following the expectation based approach. Further, a location based user selection, exploiting 
the satellite system architecture is described. As expected, it was observed that the precoder is robust against phase uncertainty and user selection improves the performance when high throughput is envisaged.

\section{ACKNOWLEDGMENT}

This work was supported by the National Research Fund, Luxembourg under AFR grant for Ph.D. project (Reference 5779106) on "Transmission and Reception Techniques for Smart Gateways in Next Generation Satellite Systems".

\section{REFERENCES}

[1] L. Cottatellucci, M. Debbah, G. Gallinaro, R. Mueller, M. Neri, and R. Rinaldo, "Interference mitigation techniques for broadband satellite systems," in 24th AIAA International Communications Satellite Systems Conference (ICSSC 2006), 2006.

[2] G. Zheng, S. Chatzinotas, and B. Ottersten, "Generic optimization of linear precoding in multibeam satellite systems," Wireless Communications, IEEE Transactions on, vol. 11, no. 6, pp. 2308-2320, 2012.

[3] D. Christopoulos, S. Chatzinotas, G. Zheng, J. Grotz, and B. Ottersten, "Linear and nonlinear techniques for multibeam joint processing in satellite communications," EURASIP journal on wireless communications and networking, vol. 2012, no. 1, pp. 1-13, 2012.

[4] ESA 6B.023, "Precoding demonstrator for broadband system forward links," ARTES 5.1 Statement of Work, 2014.

[5] A. Gharanjik, B. Shankar M. R. R., P.-D. Arapoglou, and B. Ottersten, "Multiple gateway transmit diversity in $\mathrm{q} / \mathrm{v}$ band feeder links," IEEE Transactions on Communications, vol. 63, no. 3, pp. 916-926, March 2015.

[6] A. Gharanjik, B. Shankar M. R. R., P.-D. Arapoglou, M. Bengtsson, and B. Ottersten, "Robust precoding design for multibeam downlink satellite channel with phase uncertainty," in IEEE International Conference on Acoustics, Speech and Signal Processing, ICASSP, April, 2015, pp. 3083-3087.
[7] A. Gershman, N. Sidiropoulos, S. Shahbazpanahi, M. Bengtsson, and B. Ottersten, "Convex optimization-based beamforming," IEEE Signal Processing Magazine, vol. 27, no. 3, pp. 62-75, 2010.

[8] J. Arnau and C. Mosquera, "Multiuser detection performance in multibeam satellite links under imperfect CSI," in 2012 Conference Record of the Forty Sixth Asilomar Conference on Signals, Systems and Computers (ASILOMAR), Nov. 2012, pp. 468-472.

[9] T. Maseng and P. Bakken, "A stochastic dynamic model of rain attenuation," IEEE Transactions on Communications, vol. 29, no. 5, pp. 660-669, 1981.

[10] "ITU-R Recommendation P.618-10," Propagation data and prediction method required for the design of the Earth-space telecommunication systems.

[11] A. Gharanjik, B. Shankar M. R. R., P.-D. Arapoglou, and B. Ottersten, "Gateway switching in $\mathrm{Q} / \mathrm{V}$ band satellite feeder links," IEEE Communications Letters, vol. 17, no. 7, pp. 1384-1387, 2013.

[12] W. Yu and T. Lan, "Transmitter optimization for the multi-antenna downlink with per-antenna power constraints," IEEE Transactions on Signal Processing, vol. 55, no. 6, pp. 2646-2660, 2007.

[13] S. Kandukuri and S. Boyd, "Optimal power control in interferencelimited fading wireless channels with outage-probability specifications," IEEE Transactions on Wireless Communications, vol. 1, no. 1, pp. 4655, Jan. 2002.

[14] M. Bengtsson and B. Ottersten, "Signal waveform estimation from array data in angular spread environment," in Conference Record of the Thirtieth Asilomar Conference on Signals, Systems and Computers, 1996, vol. 1, Nov. 1996, pp. 355-359 vol.1.

[15] CVX Research Inc., "CVX: Matlab software for disciplined convex programming, version 2.0," http://cvxr.com/cvx, Aug. 2012.

[16] Z.-Q. Luo, W.-K. Ma, A.-C. So, Y. Ye, and S. Zhang, "Semidefinite relaxation of quadratic optimization problems," IEEE Signal Processing Magazine, vol. 27, no. 3, pp. 20-34, 2010.

[17] M. Diaz, N. Courville, C. Mosquera, G. Liva, and G. Corazza, "Nonlinear interference mitigation for broadband multimedia satellite systems," in International Workshop on Satellite and Space Communications, 2007. IWSSC '07, pp. 61-65. 